ALS. Meanwhile, members of the ALS consortium had defined a dozen or so microsatellite markers to compile a haplotype of the $A L S$ region. One such marker, D21S223, was amplified from a cosmid in this region and showed tight linkage to ALS. Initially, it was reported that the peak lod score occurred with SOD1 at $2 \mathrm{cM}$ (suggesting at that time a significant physical separation between the two). But, says Brown, the idea of $S O D 1$ as a candidate gene resurfaced at a muscular dystrophy meeting late last year. This notion was sealed when first, D21S223 did not recombine with ALS in six families known to map to chromosome 21, and second, a portion of SODI was found in the same cosmid as D21S223, placing them just kilobases apart.

From that moment, the pieces fell into place in short order. Mutations in SOD1 were found in exons 2 and 4 in affected members of 18 ALS families, leaving no doubt as to the genetic defect and provoking interesting speculation as to the biochemical basis of the disease ${ }^{7}$. Siddique told Newsweek: "We haven't slain Goliath, but we certainly feel like a David who's been introduced to the slingshot." Quite.

SOD is an extraordinarily well studied (if not yet fully understood) enzyme about which volumes of research papers have been published during the past two decades. In simple terms, its role is to remove damaging oxygen free radicals from the cell by reducing them to hydrogen peroxide, which in turn is removed by other enzymes. SOD1 is not only found in virtually all organisms, but it is also expressed in the majority of mammalian tissues. How, then, could a putative defect in SOD1 manifest itself only in motor neurons? Could it simply be a consequence of the lack of turnover of motor neurons, or is there a more specific target of free radical damage? And how do a cluster of missense mutations in an enzyme give rise to a dominantly inherited disease, unless they somehow result in a gain of function? The fact that SOD1 exists as a homodimer provides an adequate, if unspectacular, explanation ${ }^{1}$.

In the wake of the ALS finding, interest has turned towards the two other known SODs: the mitochondrial form (Mn,SOD) which maps to chromosome 6q25, and an extracellular form that has been localized to chromosome 4 (ref. 8). Neither location corresponds to the only other known form of ALS that has been mapped - a recessive trait on chromosome 2 (ref. 9) - but they are enticing candidate genes for other disorders. Some $25 \%$ of dominantly inherited cases of ALS have still to be accounted for.

Meanwhile, work continues on unravelling another great mystery associated with ALS - the bizarre explosion of cases on the Pacific island of Guam after the Second World War ${ }^{3,4}$. The incidence of Guamanian ALS as well as outbreaks in areas of Japan and New Guinea, has been linked to the ingestion of the highly toxic cycad seeds, popular among the native population. (The disease is now on the decline, presumably due to the improved education of the general public.) Two constituents of these seeds, the amino acid $\beta-N$ methylamino-L-alanine (BMAA) and the cytotoxin cycasin, are the chief suspects. Although BMAA can produce ALS-like symptoms when injected into monkeys ${ }^{10}$, it is unlikely to be the sole explanation of Guamanian ALS. Recent work has shown that cycasin is taken up by cortical explants of neurons (possibly through the glucose transporter), leading to neurodegeneration in a matter of days ${ }^{11}$. One interesting hypothesis is that cycasin is hydrolysed in neurons to an aglycone known as methylazoxymethanol (MAM). MAM is capable of alkylating DNA, and as DNA repair is notoriously poor in nerve cells, a cumulative effect on protein synthesis might result in neurodegeneration. Perhaps free radicals affect a similar pathway.

1. Rosen, D.R. et al. Nature 362, 59-62 (1993).

2. White, M. \& Gribbin, J. Stephen Hawking - A Life in Science. (Niking/Penguin, 1992).

3. Monmaney, T. The New Yorker. October 29, 85-113 (1990).

4. Davies, K. New scientist. August 17, 21-25 (1991).

5. Siddique, T. et al. New Engl. J. Med. 324, 1381-1384 (1991).

6. Eubanks, J.H. et al. Proc. natn. Acad. Sci. U.S.A. 90, 178182 (1992).

7. McNamara, J.O. \& Fridovich, I. Nature 362, 20-21 (1993).

8. Hendrickson, D. et al. Genomics 8, 736-738 (1990).

9. Hentati, A. et al. Am. J. hum. Genet. 51 (suppi.), A33 (1992).

10. Spencer, P. et al. Science 237, 517-522 (1987).

11. Kisby, G.E. et al. Neurodegeneration 1, 73-82 (1992).

\section{Lorenzo Odone}

In Nature Genetics 3, 95-96; February 1993, Lorenzo Odone (the boy suffering from adrenoleukodystrophy who is featured in the film Lorenzo's oil) was wrongly said to be in a "vegetative state". On the contrary, his parents quote a teacher as saying that Lorenzo can see, hear and communicate by gesture and Dr Donald Fishman, the neurologist who originally diagnosed Lorenzo's condition, as saying that "the child's survival to date... must be considered marvellous". 